\title{
The Effect of Case Presentation on Student Physical Therapists' Clinical Reasoning Hypotheses
}

\author{
Nicholas LaRosa \\ University of North Florida, n.larosa@unf.edu \\ Daniel L. Dinsmore \\ University of North Florida, daniel.dinsmore@unf.edu
}

Follow this and additional works at: https://nsuworks.nova.edu/ijahsp

Part of the Education Commons, and the Medicine and Health Sciences Commons

\section{Recommended Citation}

LaRosa N, Dinsmore DL. The Effect of Case Presentation on Student Physical Therapists' Clinical Reasoning Hypotheses. The Internet Journal of Allied Health Sciences and Practice. 2021 Jan 01;19(1), Article 7.

This Manuscript is brought to you for free and open access by the College of Health Care Sciences at NSUWorks. It has been accepted for inclusion in Internet Journal of Allied Health Sciences and Practice by an authorized editor of NSUWorks. For more information, please contact nsuworks@nova.edu. 


\title{
The Effect of Case Presentation on Student Physical Therapists' Clinical Reasoning Hypotheses
}

\begin{abstract}
Purpose: Clinical reasoning is an essential skill for Physical Therapists to develop for making sound decisions regarding patient care. Case-method teaching is an instructional strategy commonly implemented in physical therapy professional education programs for facilitating clinical reasoning skill acquisition. One advantage of case-method teaching is the various ways cases can be portrayed. The purpose of this study was to identify how a case is portrayed effects student thinking and their subsequent clinical decision making. Method: Third-year student physical therapists $(n=14)$ working in dyads clinically reasoned through a hypothetical musculoskeletal case presented via written case study or simulated patient experience. Talk aloud methodology via concurrent reports was implemented for data collection. Mann-Whitney U-tests followed by manual calculations of effect sizes were conducted for comparing hypothesis category generation between groups. Results: A total of 14 hypothesis categories were generated by the student dyads during the problem-solving sessions. Specifically, students generated more ideas regarding health condition, and contextual factors when thinking through a written case study whereas significantly more thoughts regarding symptom characteristics, client perspectives, and minimizing reasoning errors were generated during simulated patient experiences. Conclusion: When implementing case-method teaching, physical therapy academic educators need to be aware that the manner a case is portrayed affects the clinical judgements students generate and their learning of clinical reasoning. Future research should continue to investigate these effects and how they ultimately impact clinical practice.
\end{abstract}

\section{Author Bio(s)}

Nicholas LaRosa, EdD, DPT, is an Assistant Professor in the Department of Clinical and Applied Movement Sciences at the University of North Florida in Jacksonville, FL. He is also a licensed physical therapist in the state of Florida.

Daniel L. Dinsmore, PhD, is an Associate Professor in the Department of Teaching, Learning and Curriculum at the University of North Florida in Jacksonville, FL. He is also the Research Director of the Northeast Florida Center for STEM Education. 


\title{
IIJAHSP \\ The Internet Joumal of Allied Health Sciences and Practice \\ Dedicated to allied health professional practice and education
}

Vol. 19 No. 1 ISSN 1540-580X

\section{The Effect of Case Presentation on Student Physical Therapists' Clinical Reasoning Hypotheses}

\author{
Nicholas LaRosa \\ Daniel L. Dinsmore \\ University of North Florida \\ United States
}

\begin{abstract}
Purpose: Clinical reasoning is an essential skill for Physical Therapists to develop for making sound decisions regarding patient care. Case-method teaching is an instructional strategy commonly implemented in physical therapy professional education programs for facilitating clinical reasoning skill acquisition. One advantage of case-method teaching is the various ways cases can be portrayed. The purpose of this study was to identify how a case is portrayed effects student thinking and their subsequent clinical decision making. Method: Third-year student physical therapists $(n=14)$ working in dyads clinically reasoned through a hypothetical musculoskeletal case presented via written case study or simulated patient experience. Talk aloud methodology via concurrent reports was implemented for data collection. Mann-Whitney U-tests followed by manual calculations of effect sizes were conducted for comparing hypothesis category generation between groups. Results: A total of 14 hypothesis categories were generated by the student dyads during the problem-solving sessions. Specifically, students generated more ideas regarding health condition, and contextual factors when thinking through a written case study whereas significantly more thoughts regarding symptom characteristics, client perspectives, and minimizing reasoning errors were generated during simulated patient experiences. Conclusion: When implementing case-method teaching, physical therapy academic educators need to be aware that the manner a case is portrayed affects the clinical judgements students generate and their learning of clinical reasoning. Future research should continue to investigate these effects and how they ultimately impact clinical practice.
\end{abstract}

Keywords: clinical reasoning, physical therapy, professional education, case-method teaching, hypothesis category 


\section{INTRODUCTION}

Physical therapy academic educators should provide valued and effective educational experiences that facilitate at minimum entrylevel skills in students. Among these skills, clinical reasoning has been identified as a foundation of professional practice and informs sound decision making. ${ }^{1}$ The central role of this type of cognitive action in both expert and novice physical therapists has been previously substantiated. ${ }^{2,3}$ Additionally, a recent concept analysis of clinical reasoning in physical therapy identified that higher order cognitive skills are requisite for successful problem-solving and decision making in clinical practice. ${ }^{4}$ Therefore, physical therapy educators need to be concerned with how the educational experiences they provide affect this type of cognitive skill growth in their students.

\section{BACKGROUND}

\section{Physical Therapist Clinical Reasoning Hypotheses}

Clinical reasoning hypotheses represent the range of cognitive judgements clinicians undertake when making decisions regarding patient care. ${ }^{5}$ Gilliland and Wainwright referred to them as any reasoning that identifies factors contributing to a patient's movement dysfunction. ${ }^{6}$ For example, physical therapists may have clinical judgements regarding health conditions that underlie their patient's pain and movement dysfunction. ${ }^{7}$ Several studies have investigated the reasoning hypotheses expert and novice physical therapists have when working with their clients. ${ }^{7-9}$ Although several hypothesis categories have been observed in physical therapists working with their clients, Jones et al stated compiling a full comprehensive list of hypothesis category judgements should not be the focus of clinical reasoning research. ${ }^{5}$ Interestingly, they also stated that reasoning hypotheses are generated in consideration of the situational context and in the manner the clinical problem unfolds. ${ }^{5}$ Therefore, an understanding of the effect of these factors on clinical reasoning hypothesis generation is warranted. Table 1 presents the range of reasoning hypotheses participants generated while working through a clinical problem. For example, students generated hypotheses regarding body structure and functioning when they considered physiological attributes such as strength and range of motion, or symptom characteristics when they considered the quantity and/or quality of the simulated "patient's" pain.

Table 1. Hypothesis Categories

\begin{tabular}{|c|c|c|}
\hline Code & Definition & Example \\
\hline Health condition & $\begin{array}{l}\text { Named pathology thought to underlie the patient's } \\
\text { condition }\end{array}$ & He could have arthritis \\
\hline Anatomical structure & $\begin{array}{l}\text { Tissues, organs, joints, and any other body part without } \\
\text { consideration for a specific health condition }\end{array}$ & $\begin{array}{l}\text { But we are more likely thinking } \\
\text { meniscus }\end{array}$ \\
\hline Body functioning/impairment & $\begin{array}{l}\text { Relating to psychosocial, physiological, or anatomical } \\
\text { structure and functioning }\end{array}$ & $\begin{array}{l}\text { Like the increased valgus at the } \\
\text { knee }\end{array}$ \\
\hline Activity/Participation & $\begin{array}{l}\text { Relating to functional movements or involvement in social } \\
\text { activities }\end{array}$ & Weekly golf outings \\
\hline Contributing factors & $\begin{array}{l}\text { Factors that develops, maintains, and/or progresses a } \\
\text { patient's problem }\end{array}$ & $\begin{array}{l}\text { So maybe he's sitting in too } \\
\text { much knee flexion }\end{array}$ \\
\hline Client perspectives & $\begin{array}{l}\text { Consideration of client's beliefs regarding their current } \\
\text { health condition and/or those factors that may be } \\
\text { contributary }\end{array}$ & $\begin{array}{l}\text { You were brought to use } \\
\text { because of pain in your left } \\
\text { knee. Is that correct? }\end{array}$ \\
\hline Symptom characteristics & $\begin{array}{l}\text { The quantity and/or quality of sensory experiences (eg, } \\
\text { pain, tingling, numbness) arising from body } \\
\text { tissues/structures }\end{array}$ & $\begin{array}{l}\text { Let me know if this makes your } \\
\text { symptoms worse. }\end{array}$ \\
\hline Mechanism of injury & $\begin{array}{l}\text { Intrinsic/extrinsic factors that caused the underlying } \\
\text { condition }\end{array}$ & $\begin{array}{l}\text { Did anything happen? Did you } \\
\text { fall? Get hit? }\end{array}$ \\
\hline Contextual factors & Attributes unique to the client & Lives in a single-story home \\
\hline Minimizing reasoning errors & $\begin{array}{l}\text { Recognition of the need for further inquiry/testing to } \\
\text { minimize potential reasoning errors }\end{array}$ & $\begin{array}{l}\text { I keep thinking we should do } \\
\text { Thessaly's later }\end{array}$ \\
\hline Phase & Consideration of the client's stage of healing & $\begin{array}{l}3 \text { months, progressive, so } \\
\text { getting worse }\end{array}$ \\
\hline $\begin{array}{l}\text { Precautions \& } \\
\text { Contraindications }\end{array}$ & $\begin{array}{l}\text { Assessment of red/yellow flag items; Consideration of the } \\
\text { need for safety tests }\end{array}$ & Looking at cardiac risk factors... \\
\hline Mgt/Tx considerations & $\begin{array}{l}\text { Health management considerations including need for } \\
\text { referral to another practitioner, client advocacy, and/or } \\
\text { prescribing procedural interventions }\end{array}$ & $\begin{array}{l}\text { Maybe we should refer to the } \\
\text { primary care physician }\end{array}$ \\
\hline
\end{tabular}




\section{Pedagogy of Clinical Reasoning in Physical Therapy Education}

A recent survey of physical therapist professional education programs revealed instruction in clinical reasoning is incorporated in all curricula, but significant variety exists in the execution. ${ }^{10}$ For example, many programs use frameworks such as the Guide to Physical Therapy Practice Patient/Client Model and the International Classification of Functioning and Disability and Health (ICF model). ${ }^{11,12}$ However, other teaching strategies like experiential learning experiences, use of the Hypothesis-Oriented Algorithm for Clinicians (HOAC), evidence-based practice, problem-based learning, and separate clinical reasoning courses are also used. ${ }^{10}$ Many of these strategies have been empirically studied for their effectiveness in facilitating clinical reasoning skill acquisition in students. For instance, use of the HOAC was found to facilitate the decision-making capabilities of students in a case involving limited ankle dorsiflexion motion impacting sit-to-stand transfers. ${ }^{13}$

Additionally, case-method teaching has been recognized as a viable instructional method to facilitate desired clinical reasoning skills. ${ }^{14}$ Case method teaching involves using case reports - often times derived from real life situations - to actively engage the student in problem-solving, and decision-making. ${ }^{15}$ One advantage of implementing case-method teaching is the various ways cases can be created and presented to students. ${ }^{16}$ Two common case presentation methods investigated in the literature are written case studies and simulated patient experiences. $13,17,18$ Written case studies are narratives that provide clinical data to students at the educator's discretion. Some advantages of written case studies include being relatively cheap to produce and easily modeled after published case reports. ${ }^{19}$ On the other hand, simulated patient experiences use trained actors to portray clinical cases. Working with simulated patients has been shown provide a higher sense of realism by mirroring the interactions students can expect to encounter during their clinical education experiences. ${ }^{20}$ Unlike written case reports which provide clinical data sequentially and at predetermined time frames, simulated patient experiences unfold in the sequence and rate concurrent with student performance of procedures. These differences may affect the cognitive reasoning of students working through the clinical problem. We know of only one study that investigated the effect of case presentation type on reasoning skill acquisition in student physical therapists. In that study, Huhn et al identified no significant differences in critical thinking between large group discussion and virtual patient experiences. ${ }^{17}$ However, we know of no study that has investigated the effect case-method presentation type has on the clinical reasoning hypotheses generated by student physical therapists.

With a focus on facilitating sound cognitive skills and an understanding that the context effects cognitive processing, we sought to investigate the effect of case-method problem presentation on the clinical reasoning hypotheses generated by student physical therapists. Specifically, our hypothesis was that students would generate different hypotheses to guide their clinical reasoning depending on how a clinical case was presented - either as a written case study or simulated patient experience. This investigation received ethical approval (IRB No. 1257814-2) prior to participant recruitment.

\section{METHODOLOGY \\ Overview of Methods}

This study sought to understand the effect case method presentation - written case study and simulated patient experience - on the clinical judgements student physical therapists generate when working through the same clinical problem. First, a hypothetical musculoskeletal case of a person with knee osteoarthritis was developed by three physical therapists including the primary investigator. Each therapist was a board-certified clinical specialist in orthopaedics and graduated from advanced residency and fellowship training programs. After creating the clinical case, each therapist verified it portrayed a typical clinical presentation for an individual with knee osteoarthritis.

To ensure the case scenario was portrayed accurately across all simulated patient encounters, one of the content experts who assisted in creating the clinical case served as the actor. ${ }^{21}$ Second, the primary investigator verified all clinical data was communicated clearly and accurately across the problem-solving sessions.

Think-aloud methodology via concurrent verbal reports was implemented for making internalized thoughts explicit during the problem-solving sessions. To ensure participants were comfortable providing concurrent verbal reports each dyad practiced thinking out loud while solving a novel puzzle prior to each problem-solving session. ${ }^{22}$ All problem-solving sessions began with presentation of general background information including the age, weight, height, and reason for referral to physical therapy (i.e., anterior knee pain). Participants assigned to the simulated patient experience then conducted a subjective and objective examination of the simulated patient for collecting clinical data and determining a diagnosis. Conversely, participants working through the written case study were provided clinical data in sequential order beginning with subjective data followed by portions of objective findings. ${ }^{19}$ New clinical data was provided every 5 minutes or sooner if participants requested it. Participants in the written case study group were instructed to speak regarding their thoughts regarding the case findings and not to read from the case study. However, when this did occur, the primary investigator provided simple reminders to focus their verbalizations on their current thinking. Each session was limited to 30 minutes in length. This length of time was chosen because it is typical of the

(C) The Internet Journal of Allied Health Sciences and Practice, 2021 
amount of time a clinician would have for an initial evaluation of a new patient. Each problem-solving session was audio-video recorded. Verbatim transcriptions of each session were created and verified for accuracy. Manual tabulations of hypothesis code frequency were made for data analysis.

\section{Participants}

The nature of the clinical case itself and the need for the participants to conduct physical examination tests and measures on an actor were both considerations when selecting participants. Third year students in their final semester of academic course work from a southeast Doctor of Physical Therapy program were recruited for the study. These students had completed all required musculoskeletal course work in the DPT curriculum while having already completed two full-time clinical education experiences. As a result of these experiences, it was felt they would be best suited for working through the problem-solving sessions. A total of 14 students agreed to participate in the study. First, study participants were randomly placed into dyads. Following random assignment, the primary investigator allocated each dyad pair into either a simulated patient or written case study group. The primary investigator conducted this step without knowledge of which participants were assigned to each group to minimize the potential for allocation bias. Dyads were used because prior research has found that students produce significantly more reasoning hypotheses when working in pairs than alone. ${ }^{23}$ Therefore, we believed dyads would provide the richest data set. A total of three dyads completed the simulated patient experience whereas four dyads completed the written case study problem solving session.

\section{Data Analysis}

An iterative process of data analysis was implemented for coding the verbatim transcriptions. The primary investigator was responsible for coding the verbatim transcriptions. This process relied on multiple sources including student hand-written notes, the primary investigator's observations of participant interactions, and studies that have already described the hypotheses physical therapists generate in their reasoning. ${ }^{5-7,23}$ Recently, Jones et al have stated that our goal in understanding clinical reasoning shouldn't be to compile a comprehensive list of all the clinical hypotheses therapists make. ${ }^{5}$ Therefore, when crafting the finalized coding scheme, we chose to rely on hypothesis categories already observed in prior studies. Within each problem-solving session, participants made several references to the same hypothesis category. That is, participants may have considered knee range of motion multiple times in their reasoning. The primary investigator decided to count each duplicate reference as separate instances when they were considered distinctly apart from each other. For instance, one dyad in the simulated patient group decided they wanted to measure the amount of hip strength the "patient" had. Considering and performing hip strength testing under these circumstances was considered a hypothesis of body functioning/impairment, however later in the same problem-solving session the dyad considered the finding of hip weakness as a possible contributing factor to the presenting knee pain. Under this condition hip weakness was coded as a contributing factor. Statistical software, IBM SPSS Statistics Version 25.0.0.1, was used for running statistical analysis. Comparisons among hypothesis categories were made using Mann-Whitney $U$ testing. Hypothesis category generation between groups was considered to significantly differ at $p<.05$. Additionally, effect sizes were manually calculated using the following equation: $\eta^{2}=Z^{2} / n$ to further understand the magnitude of the effect case problem presentation method has on student's clinical judgements. ${ }^{24}$

\section{Reliability}

Several measures were taken to enhance credibility and trustworthiness of the study findings. Data triangulation was achieved using verbatim transcriptions, student notes, and the direct observations of the primary investigator of the study. The primary investigator considered these data collectively when applying hypothesis category codes to participant verbalizations made during the problem-solving sessions. To enhance credibility of the study findings a subsample of data was coded by another physical therapist with over 30 years' experience as a practicing clinician and has taught in accredited physical therapy professional education programs, including clinical reasoning, for the past 16 years. Peer-debriefing was implemented to resolve any differences in opinion regarding the hypotheses code definitions and their application to the verbatim transcriptions. This process continued until an inter-rater reliability of $\mathrm{k}=.714$ was achieved indicating substantial agreement for hypothesis category code application to the verbatim transcriptions. ${ }^{25}$

\section{RESULTS}

\section{Demographic Comparisons}

Upon visual inspection it was determined each group was homogenous regarding age, GPA, race, and ethnicity. However, it was noted that the groups appeared to differ based on gender and area of interest in physical therapy practice at the time of the study (Table 2). 
Table 2. Participant Description

\begin{tabular}{lcc}
\hline \multicolumn{1}{c}{ Characteristic } & Simulated Patient & Written Case Study \\
\hline Age (years) & $25.50(1.38)$ & $26.13(2.64)$ \\
\hline GPA $^{\mathrm{a}}$ & $3.73(.11)^{\mathrm{b}}$ & $3.64(.25)$ \\
\hline Female gender & $66.66 \%$ & $25 \%$ \\
\hline Male gender & $33.33 \%$ & $75 \%$ \\
\hline Race (White) & $100 \%$ & $100 \%^{\mathrm{b}}$ \\
\hline $\begin{array}{l}\text { Ethnicity (Not Hispanic or } \\
\text { Latino) }\end{array}$ & $100 \%$ & $100 \%^{\mathrm{b}}$ \\
\hline Interest (orthopaedics) & $50 \%$ & $87.5 \%$ \\
\hline
\end{tabular}

a Values expressed as mean (SD)

${ }^{b}$ Information not provided by one participant

\section{Hypothesis Category Comparisons}

A total of 13 hypothesis categories were generated by student participants during the problem-solving sessions (Table 1). These hypothesis categories were created in consideration of previous investigations into the clinical reasoning of expert and novice physical therapists ${ }^{5-7,23,26}$ and represent the entire range of clinical reasoning hypotheses student participants generated during the problem-solving sessions.

Quantitative analysis of the frequency of hypothesis category generation for each group was conducted. Mean values with standard deviations were calculated followed by Mann Whitney- $U$ comparisons (Table 3). These data suggest the clinical judgements student physical therapists had was affected by case presentation type. Specifically, the hypothesis categories health condition, symptom characteristics, and contextual factors were generated significantly more by dyads assigned to the written case study condition. Additionally, the hypothesis categories client perspectives and minimizing reasoning errors were considered significantly more by dyads in the simulated patient group. Manual calculations identified moderate-to-large effect sizes $\left(\eta^{2}=.64-.71\right)$ for each of these hypothesis categories. These values imply case presentation type was mostly responsible for the differences in the frequency of hypothesis category generation between groups.

Table 3. Comparing Reasoning Hypothesis Generation

\begin{tabular}{lccc}
\hline \multicolumn{1}{c}{ Code } & $\begin{array}{c}\text { Simulated } \\
\text { Patienta }\end{array}$ & Written Case Studya & $\begin{array}{c}\text { Mean } \\
\text { Difference }\left(\eta^{2}\right)\end{array}$ \\
\hline Health condition & $7.33(4.51)$ & $14.5(1.00)$ & $\mathbf{7 . 1 7}(.69)$ \\
\hline Anatomical structure & $19.33(10.69)$ & $22.50(5.45)$ & $3.17(.02)$ \\
\hline Body functioning/impairment & $46.33(14.57)$ & $32.50(9.00)$ & $13.83(.22)$ \\
\hline Activity/participation & $4.67(3.22)$ & $10.75(4.57)$ & $6.08(.37)$ \\
\hline Contributing factors & $29.33(8.39)$ & $22.5(4.36)$ & $6.83(.07)$ \\
\hline Client perspectives & $2.67(.58)$ & $.25(.50)$ & $\mathbf{2 . 2 4}(.71)$ \\
\hline Symptom characteristics & $28.67(4.73)$ & $17.00(4.97)$ & $\mathbf{1 1 . 6 7 ( . 6 4 )}$ \\
\hline Mechanism of injury & $3.33(1.53)$ & $4.75(2.63)$ & $1.42(.08)$ \\
\hline Contextual factors & $4.67(.58)$ & $9.00(1.83)$ & $4.33(.70)$ \\
\hline Minimizing reasoning errors & $18.33(9.24)$ & $2.00(1.83)$ & $16.33(.65)$ \\
\hline Phase & $3.00(1.73)$ & $2.50(1.00)$ & $.50(.03)$ \\
\hline Precautions \& contraindications & $5.33(8.39)$ & $3.00(2.94)$ & $2.33(<.00)$ \\
\hline Mgt/Tx Considerations & $2.00(1.73)$ & $6.00(4.69)$ & $4.00(.30)$ \\
\hline
\end{tabular}

a Values are expressed as mean (SD)

Mean differences in bold are statistically significant at $p<.05$

\section{DISCUSSION}

The study results support the idea that the situational context in which clinical reasoning takes place significantly effects the clinical judgements student physical therapists make when problem-solving. One reason for this could be the inherent nature of the case study presentation methods themselves. It has been stated elsewhere that varied case presentation methods allow flexibility in the content of clinical cases presented and the manner they unfold. ${ }^{16,19} \mathrm{In}$ this study, dyads assigned to the simulated patient group needed to actively acquire clinical data through subjective inquiry and performance of selected physical tests. This led to significantly more time on considering the need for further testing, minimizing reasoning error, and limiting the breadth of possible 
alternative diagnoses, health conditions. For instance, two out of three simulated patient dyads did not perform a cardiovascular assessment of the actor as part of their physical examination. Therefore, they did not receive clinical data regarding blood pressure, heart rate, and respiratory rate. Conversely, every written case study dyad was provided this data which facilitated the consideration of alternative health conditions such as hypertension, and cardiovascular disease.

Regarding contextual factors and symptom characteristics, we identified significant differences between groups. Specifically, written case study dyads considered contextual factors like work environment and recreational activities at nearly double the frequency while simulated patient dyads considered significantly more symptom characteristics. Although we identified all groups considered contextual factors in their diagnostic reasoning, written case study dyads who were provided all relevant contextual factors of the case seemed to weigh their impact more by linking clinical signs and symptoms to these factors whereas simulated patient dyads mostly downplayed their contribution to the clinical case. Previously, Edwards et al described the dialectical nature of clinical reasoning in expert physical therapists. ${ }^{27}$ They identified expert therapists consider both scientifically grounded empiricoanalytical thinking with more subjectively grounded narrative reasoning. We identified written case study dyads similarly used both forms of thinking in their diagnostic reasoning. Conversely, simulated patient dyads relied primarily on empirico-analytical thinking as evidence by heavily considering how objective tests and measures affected the quantity and quality of the "patient's" pain experiences (i.e., symptom characteristics) in their diagnostic reasoning.

Effect size calculations for statistically significant differences in hypotheses made between groups were identified as moderate-tolarge $\left(\eta^{2}=.64-.71\right)$. This is an important finding because it means case presentation method greatly influenced the clinical hypotheses student participants considered. For instance, we identified a mean difference of 4.33 instances of contextual factor hypotheses generated between groups. The effect size for this hypothesis category was $\eta^{2}=.70$, meaning case presentation method accounted for $70 \%$ of the variance in the generation of hypotheses regarding contextual factors for the case in this sample. These findings indicate that case presentation method significantly influenced the kinds of hypotheses student participants had in their clinical reasoning. Physical therapy educators can use this knowledge when constructing clinical case scenarios for their students in the classroom to promote desired thinking and reasoning.

Effect sizes should be interpreted cautiously in studies with small sample sizes because of the increased probability of making a Type II error. ${ }^{24}$ Therefore, effect size estimates in research with small sample sizes should only be considered when groups are found to statistically differ from each other. Therefore, while the calculated effect size for the hypothesis category activity/participation was $\eta^{2}=.37$, research with larger sample sizes should be conducted before drawing any meaningful conclusions from this finding.

The results of this research may have significant implications for physical therapy education. It has been suggested that simulated patient experiences may be more valuable than written case studies because they more closely mirror actual clinical experiences students can expect to have during the clinical education portions of their respective professional education programs, and facilitate non-reasoning skills like conducting subjective interviews. ${ }^{19}$ However, we identified written case study facilitated the consideration of contextual factors more than simulated patient experiences. Recently, Jones et al has suggested that physical therapy clinical reasoning be situated in a biopsychosocial framework. ${ }^{5}$ Specifically, they suggested use of the ICF model for making explicit the impact contextual factors like environment and personal factors have on the health and functioning of individuals. Although all study participants received similar educational experiences regarding use of the ICF model when working with clients, we identified written case study method promoted a biopsychosocial approach to client management, a desired trait expert therapists often exhibit. $3-5,27$ The results of our investigation have determined that the way a clinical case is portrayed - written case study or simulated patient experience -emphasizes different thinking and hypothesis formation in student physical therapists. Physical therapy academic educators can use this knowledge to assist creating educational experiences that facilitate desired physical therapy specific clinical reasoning skills.

\section{Limitations}

This study had a few limitations that warrant discussion. First, the clinical case represented a "typical" presentation of a person seeking physical therapy for anterior knee pain. However, reasoning in physical therapy has been found to differ dependent on factors such as clinical environment and patient population served. ${ }^{28,29}$ Future research should explore the effects of case method pedagogy on the clinical reasoning hypotheses generated by students across varied patient populations. Second, the small and homogenous sample prohibits generalization of the study findings to the larger population. However, we do believe the study findings provide context for physical therapy academic educators when choosing to implement case-method teaching in the classroom. Third, there were multiple potential sources of bias identified. For example, the use of only one coder for the verbatim transcriptions and relying on previously observed hypothesis categories could have biased the results and findings of the study. Furthermore, the coder attempted to consider duplicate hypothesis categories distinctly apart from each other when the same

(C) The Internet Journal of Allied Health Sciences and Practice, 2021 
hypothesis was used under different contexts. Although this strategy was implemented to reduce inflation of hypothesis category counts, the use of one coder restricts confirming this assertion. Lastly, participants worked together in dyads during the problemsolving sessions. Typically, physical therapy clinicians perform patient examinations alone without the assistance of another therapist. However, as stated previously we sought to collect the richest data set possible for ascertaining the range of clinical judgements students have while problem-solving through the clinical case. Additionally, students often work alongside a clinical instructor during their clinical education experiences and can therefore expect to work with a partner when conducting patient examinations during these experiences. Lastly, we identified potential heterogeneity among groups regarding gender and interest in orthopaedic physical therapy. Although, these differences existed, we believe the small sample size exaggerates their meaning and did not significantly impact the study findings.

\section{Future Directions}

While this research focused on the quantity of the reasoning processes utilized, it would also be important to examine both the quality of those strategies (i.e., how well they were implemented) and the conditional use of those processes (i.e., under what conditions they were utilized) to gain a fuller picture of the relations between clinical reasoning and performance. ${ }^{30}$ While there has been a demonstrated relation between the quantity of a strategy - in this case the reasoning processes - knowing how well or when students utilize these processes would help us better understand how these different aspects of strategy use influences those outcomes. This will likely necessitate the use of follow-up interview protocols and new frameworks for assessing the quality of these reasoning processes, a promising next step for this research.

\section{CONCLUSION}

Case-method teaching is a commonly implemented instructional strategy PT educators implement with their students. Several methods for case study presentation type have been identified in the literature. This is the first study that we know that examined the effect case presentation type has on the clinical judgments student physical therapist generate in their diagnostic clinical reasoning. Future research should continue to consider how educational experiences influence student physical therapist hypothesis formation, their learning of physical therapy specific clinical reasoning, and ultimately how it impacts their clinical practice.

\section{REFERENCES}

1. Higgs J, Jensen GM. Clinical Reasoning: Challenges of Interpretation and Practice in the 21st Century. In: Higgs J, Jensen GM, Loftus S, Christensen N, eds. Clinical Reasoning in the Health Professions. 4th ed. Edinburgh: Elsevier; 2019:3-11.

2. Furze J, Black L, Hoffman J, Barr JB, Cochran TM, Jensen GM. Exploration of Students' Clinical Reasoning Development in Professional Physical Therapy Education. Journal of Physical Therapy Education. 2015;29(3):22-33.

3. Doody C, McAteer M. Clinical reasoning of expert and novice physiotherapists in an outpatient orthopaedic setting. Physiotherapy. 2002;88(5):258-268. doi: 10.1016/S0031-9406(05)61417-4

4. Huhn K, Gilliland SJ, Black LL, Wainwright SF, Christensen N. Clinical Reasoning in Physical Therapy: A Concept Analysis. Phys Ther. 2019;99(4):440-456. doi: https://doi.org/10.1093/ptj/pzy148 [PMID 30496522]

5. Jones MA, Edwards I, Jensen GM. Clinical reasoning in physiotherapy. In: Higgs J, Jensen GM, Loftus S, Christensen $\mathrm{N}$, eds. Clinical Reasoning in the Health Professions. 4th ed. Edinburgh: Elsevier; 2019:247-260.

6. Gilliland S, Wainwright SF. Patterns of Clinical Reasoning in Physical Therapist Students. Phys Ther. 2017;97(5):499511. doi: 10.1093/pt/pzx028 [PMID 28371873]

7. Gilliland S. Physical Therapist Students' Development of Diagnostic Reasoning: A Longitudinal Study. Journal of Physical Therapy Education. 2017;31(1):31-48. doi: 10.1097/00001416-201731010-00007

8. Jones MA. Clinical reasoning: from the Maitland Concept and beyond. In: Hengeveld E, Banks K, eds. Maitland's vertebral manipulation: Management of neuromusculoskeletal disorders. Vol 1. 8th ed. Edinburgh, Scotland: Churchill Livingstone Elsevier; 2014:14-82.

9. Jones MA, Rivett DA. Introduction to clinical reasoning. In: Jones MA, Rivett DA, eds. Clinical Reasoning for Manual Therapists. Edinburgh: Elsevier; 2004:3-24.

10. Christensen N, Black L, Furze J, Huhn K, Vendrely A, Wainwright S. Clinical Reasoning: Survey of Teaching Methods, Integration, and Assessment in Entry-Level Physical Therapist Academic Education. Phys Ther. 2017;97(2):175-186. [PMID 27609900]

11. American Physical Therapy Association. Guide to Physical Therapist Practice 3.0. 2014. http://guidetoptpractice.apta.org/. Accessed June 30, 2017. 
12. World Health Organization. International Classification of Functioning, Disability and Health. Geneva: World Health Organization; 2001.

13. Gillardon P, Zipp GP. A Proposed Strategy to Facilitate Clinical Decision Making in Physical Therapist Students. Journal of Physical Therapy Education. 2002;16(2):57-63.

14. Patton N, Christensen N. Pedagogies for teaching and learning clinical reasoning. In: Higgs J, Jensen GM, Loftus S, Christensen N, eds. Clinical Reasoning in the Health Professions. 4th ed. Edinburgh: Elsevier; 2019:335-344.

15. Jensen GM, Mostrom E, Shepard KF. Teaching and learning in academic settings. In: Jensen GM, Mostrom E, eds. Handbook of Teaching and Learning for Physical Therapists. 3rd ed. St. Louis, MO: Elsevier; 2013:36-62.

16. McGinty SM. Case-method teaching: an overview of the pedagogy and rationale for its use in physical therapy education. Journal of Physical Therapy Education. 2000;14(1):48-51.

17. Huhn K, McGinnis PQ, Wainwright S, Deutsch JE. A Comparison of 2 Case Delivery Methods: Virtual and Live. Journal of Physical Therapy Education. 2013;27(3):41-48. doi: [PMID

18. Miller AH, Tomlinson S, Tomlinson JD, Readinger J. Addition of a Patient Examination Module to Address Student Preparedness for the First Full-Time Clinical Experience. Journal of Physical Therapy Education. 2017;31(2):30-43. doi: 10.1097/00001416-201731020-00005 [PMID

19. Rivett DA, Jones MA. Using case reports to teach clinical reasoning. In: Higgs J, Jones MA, Loftus S, Christensen N, eds. Clinical Reasoning in the Health Professions. 3rd ed. Amsterdam: Elsevier; 2008:477-484.

20. Ladyshewsky RK, Baker R, Jones M, Nelson L. Evaluating clinical performance in physical therapy with simulated patients. Journal of Physical Therapy Education. 2000;14(1):31-37.

21. Tamblyn RM, Klass DJ, Schnabl GK, Kopelow ML. The accuracy of standardized patient presentation. Med Educ. 1991;25(2):100-109. doi: 10.1111/j.1365-2923.1991.tb00035.x [PMID 2023551]

22. Ericsson KA, Simon HA. Protocol Analysis : Verbal Reports As Data. Rev. ed. Cambridge, Mass: MIT Press; 1992.

23. Ladyshewsky RK. The Impact of Peer-Coaching on the Clinical Reasoning of the Novice Practitioner. Physiother Can. 2004;56(1):15-25.

24. Tomczak M, Tomczak EWA. The need to report effect size estimates revisited. An overview of some recommended measures of effect size. Trends in Sport Sciences. 2014;21(1):19-25.

25. Hallgren KA. Computing Inter-Rater Reliability for Observational Data: An Overview and Tutorial. Tutorials in Quantitative Methods for Psychology. 2012;8(1):23-34. doi: [PMID 22833776]

26. Gilliand S. Clinical Reasoning in First- and Third-Year Physical Therapist Students. Journal of Physical Therapy Education. 2014;28(3):64-80.

27. Edwards I, Jones M, Carr J, Braunack-Mayer A, Jensen GM. Clinical reasoning strategies in physical therapy. Phys Ther. 2004;84(4):312-335. [PMID 15049726]

28. Masley PM, Havrilko C-L, Mahnensmith MR, Aubert M, Jette DU, Coffin-Zadai C. Physical Therapist Practice in the Acute Care Setting: A Qualitative Study. Phys Ther. 2011;91(6):906-922. doi: 10.2522/ptj.20100296 [PMID 21511991]

29. Kenyon LK. The Hypothesis-Oriented Pediatric Focused Algorithm: A Framework for Clinical Reasoning in Pediatric Physical Therapist Practice. Phys Ther. 2013;93(3):412-421. doi: 10.2522/ptj.20120080 [PMID 23086410]

30. Dinsmore DL. Towards a dynamic, multidimensional model of strategic processing. Educ Psychol Rev. 2017;29:235268. doi: 10.1007/s10648-017-9407-5 